\title{
FDG-PET/CT in predicting aggressiveness of rectal cancer
}

\author{
Iman Sherif Ahmed, Saher Mohamed El Gaafary, Remon Zaher Elia and Rasha S. Hussein * (]
}

\begin{abstract}
Background: Treatment response varies significantly among rectal cancer patients. Tumor can show complete regression, stationary appearance, or even tumour progression during the treatment. It is also widely known that the rate of local recurrence is variable. Precise risk stratification of tumor aggressiveness is required for better per patient tailored treatment plan and predicting the overall prognosis of rectal cancer patients The aim of this study was to assess different parameters of baseline [18F] fluorodeoxyglucose positron emission tomography/computed tomography [(18F) FDG-PET/CT] as a non-invasive tool in predicting aggressiveness of the rectal cancer.

Results: Overall, 33 patients were included [19 moderately differentiated adenocarcinoma, 10 poorly differentiated adenocarcinoma and 4 mucinous adenocarcinomas (MAC)]. SUV estimates (SUV max, SUV mean) were greater in the moderately adenocarcinoma group ( $p=0.003$ and $p=0.019$, respectively). MTV and TLG values were similar between the three histopathological groups ( $p=0.763$ and $p=0.701$, respectively). There was no correlation between SUVmax of primary tumor and MTV $(r=0.034 ; p=0.849)$. However, SUVmax and TLG were significantly correlated $(r=0.517$; $p=0.002)$. Strong correlation between tumor size and MTV $(r=0.489 ; p=0.003)$, and TLG $(r=0.506 ; p=0.003)$ were observed. No significant association was found between MTV and TLG and the clinical stage of rectal cancer.

Conclusion: Baseline 18F-FDG PET/CT parameters cannot be used alone as a non-invasive diagnostic technique in assessing aggressiveness and prognosis in patients with primary rectal cancer, and further clinical studies are needed before considering the prognostic role of FDG-PET/CT in rectal cancer.
\end{abstract}

Keywords: FDG-PET/CT, Aggressiveness assessment, Rectal cancer, MTV, TLG

\section{Background}

Colorectal cancer [CRC] is the world's third most frequent malignancy and the second leading cause of cancer-related death [1]. Treatment response varies significantly among rectal cancer patients. Tumor can show complete regression, stationary appearance, or even tumour progression during the treatment. It is also widely known that the rate of local recurrence is variable $[2,3]$.

In this context, precise risk stratification is required for better per patient tailored treatment plan, assessment of tumor aggressiveness and predicting the overall

*Correspondence: rashasalah@med.asu.edu.eg; rashasalaheldein@gmail.com Radiology Department, Faculty of Medicine, Ain Shams University, Cairo 11566, Egypt prognosis of rectal cancer patients. Patient age, tumour size, primary tumour localization, lymphovascular invasion, perineural invasion, initial lymph node metastasis, stage, localization of metastasis, and tumour differentiation are well-known predefined clinic-pathological risk factors for aggressive CRC [4].

[18F] fluorodeoxyglucose positron emission tomography/computed tomography [(18F) FDG-PET/CT] is a type of imaging that detects and quantifies metabolic avidity in cancer cells, acting as a surrogate for cellular activity and vitality [5]. FDG accumulation in tumour cells is based on increased glycol-metabolism, and there is strong evidence that FDG uptake in tumour cells is linked to cancer growth rate, aggressiveness, and prognosis [6]. Several 18F-FDG PET/CT parameters, including SUVmax, SUVmean, MTV, and Total Lesion Glycolysis 
TLG, have been proposed as useful prognostic indicators for many tumors $[7,8]$. Volumetric parameters, such as MTV and TLG, are expected to aid in measuring volumetric tumour burden and serve as prognostic factors in malignant disease [9].

Some studies in rectal cancer reported that the MTV and TLG are validated as strong pretreatment prognostic factors for cancer recurrence and cancer-related death [10]. Patients with a high MTV or TLG had a worse clinical outcome than those with a low MTV or TLG. Another study concluded that higher SUVmax is significantly associated with shorter survival in patients with colorectal cancer, and that SUVmax is a useful preoperative prognostic factor in these patients [11]. On the contrary, another study reported that high FDG uptake of primary mass in resectable colorectal cancer does not have a significant relationship with tumor recurrence and disease-free survival [12].

There is insufficient information in these published studies regarding whether the metabolic parameters of rectal cancer lesions are related to conventional risk factors of cancer aggressive behavior (such as high tumor stage, large size, and some histological subtypes). We designed the present study to assess the correlation between the aggressiveness as determined by some conventional risk factors and glucose metabolism as measured by FDG PET/CT in rectal adenocarcinoma.

\section{Methods}

\section{Patient population}

Thirty-three patients with histologically proven rectal cancer were investigated by FDG-PET/CT between March 2019 and May 2021, prior to the initiation of treatment. Inclusion criteria were biopsy-proven rectal cancer, patients' age $>18$ years, and absence of other concomitant tumors. Exclusion criteria were high serum creatinine level $(>1.5 \mathrm{mg} / \mathrm{dl})$ and previous anticancer therapy. The study was performed after approval of the Ethical Committee of Scientific Research, Faculty of Medicine, Ain Shams University. Consent was taken from all patients. Histopathologic subtypes were collected from each patient.

\section{$\mathrm{PET} / \mathrm{CT}$}

Patients fasted for at least six hours before intravenous administration of radioactive tracer FDG isotope with the dose of $0.06-0.08 \mathrm{mci} / \mathrm{kg}$. Pre-injection blood glucose levels were measured to make sure that they were below $200 \mathrm{mg} / \mathrm{dl}$. During the distribution phase, the patients laid supine in a quiet room. PET/CT study was performed using a dedicated hybrid PET/CT scanner GE medical system [(GE discovery IQ 5 rings) and enhanced helical CT (optima 540 16-slice)]. Combined image acquisition began 60 min after FDG injection. The patient was positioned supine on the table. Single-phase contrast material-enhanced helical CT using a standardized protocol [28-30 mAs; $120 \mathrm{kV}$; slice thickness $5 \mathrm{~mm}$ ] was performed following injection of $125 \mathrm{~mL}$ of a low osmolarity iodinated contrast medium (Optiray 350 ) at a rate of $4 \mathrm{~mL} / \mathrm{sec}$ by using a power injector. A whole-body CT study [neck, chest, abdomen, and pelvis] scanning was obtained. The corresponding PET imaging immediately followed over the same body region without repositioning the patient on the table. Six to seven-bed positions were planned in the three-dimensional acquisition mode for scanning the entire patient with 5-7 min acquisition at each bed position. PET images were acquired with shallow breathing. Attenuation was corrected using the $\mathrm{CT}$ images and the images were reconstructed. Delayed PET CT images were needed in some cases if the uptake of the lesion is controversial.

\section{Image analysis}

Images were transferred to the offline GE workstation (Advantage window 4.7). Fused images were obtained. Two experienced nuclear medicine physicians with 7 and 5 years' experience (SM and RS respectively) interpreted $\mathrm{PET} / \mathrm{CT}$ images and a final consensus was reached for all the patients. The tumor size was the maximum length measured on CT images. Rectal mass was categorized based on location, size, and visual correlation between CT and PET images to differentiate physiologic uptake from pathologic uptake. A lymph node was considered PET-positive if its FDG uptake was higher than blood pool activity or surrounding background tissue. A volumetric region of interest (ROI) around the outline of the primary tumor was placed on the axial 18F-FDG PET/ CT images using semi-automatic software. The ROI borders were manually adjusted by visual inspection of the primary tumor to avoid an overlap of adjacent FDG-avid structures. The 18F-FDG uptake of the urinary tract and bladder was excluded. For each 18F-FDG PET/CT study, the SUVmax, SUVmean, MTV, and TLG values of the primary tumor were measured. The PET parameters, including SUVmean, SUVmax, MTV, and TLG were automatically acquired.

\section{Statistical analysis}

Analysis of data was done using SPSS (Statistical package for social science) program version 23 . To describe the studied sample, quantitative data were presented as median \& range. Qualitative data were presented as count \& percentage. The Chi square statistic is used for testing relationships between categorical variables. The Wilcoxon signed-rank test is a non-parametric statistical hypothesis test used to compare two related samples, 
matched samples, or repeated measurements on a single sample to assess whether their population mean ranks differ (i.e. it is a paired difference test). One-Way ANOVA test was used to compare parametric quantitative data between more than two groups. Kruskal-Wallis test was used to compare nonparametric quantitative data between different groups.

\section{Results}

\section{Patient and tumor characteristics}

Overall, 33 patients, who had sufficient histological information, were included in the study. The median age of the patients was 60 years (range 32-36 years), and 19 of these patients (57.57\%) were men. Four patients (12.1\%) were classified as MAC, 19 (57.6\%) as moderately differentiated adenocarcinoma, and $10(30.3 \%)$ as poorly differentiated adenocarcinoma. All primary rectal tumors could be visually identified on PET/CT images regardless of the histological subtype, resulting in a $0 \%$ falsenegative rate. Ten patients (30.3\%) had clinical stage IIA disease, six patients (18.2\%) had clinical stage III disease, and seventeen patients (51.5\%) had clinical stage IV disease (Table 1).

\section{8-F FDG PET/CT findings}

The median and range values of SUVmax, SUVmean, MTV and TLG values were 17.9 (5.3-53.82), 10.46 (2.740.5), $21.5(3.9-62.5) \mathrm{cm}^{3}$ and $234.442(36.9-2521.1) \mathrm{g} /$ $\mathrm{ml}^{* *} \mathrm{xcm}^{3}$ for the entire group, respectively. There was no correlation between the SUVmax of the primary rectal tumor and MTV ( $p$ value was 0.849 ). Whereas the correlation between SUVmax and TLG was highly significant ( $p$ value was 0.002 ) (Fig. 1).

\section{Correlations between FDG-PET/CT parameters and patient/ tumor characteristics}

The demographic and PET/CT characteristics according to the different histopathological groups are presented in Table 2. Median tumor sizes were similar between the three groups $(p=0.765)$. Median value of SUVmax of primary tumors of poorly differentiated, moderately differentiated adenocarcinoma, and mucinous adenocarcinoma patients were calculated as 14.55 versus 22.3 , and 12.68 respectively. The difference between groups was highly significant $(p<0.01)$. The median value of SUVmean were statistically greater in the moderately differentiated adenocarcinoma group than poorly differentiated and mucinous adenocarcinoma (13.8 vs. 8.31 , and 8.4 respectively). The difference between groups was statistically significant $(p<0.05)$.

There were no significant differences between MAC, moderately differentiated adenocarcinoma, and poorly differentiated groups as regarding the median values of
Table 1 Patient and tumor characteristics

\begin{tabular}{|c|c|}
\hline Characteristic & Number [\%] \\
\hline Age median, range & 60 [32-86] \\
\hline \multicolumn{2}{|l|}{ Sex } \\
\hline Male & 19 [57.57\%] \\
\hline Female & $14[42.42 \%]$ \\
\hline \multicolumn{2}{|l|}{ Location } \\
\hline Upper rectum & 19 [57.6\%] \\
\hline Middle rectum & $3[9.1 \%]$ \\
\hline Lower rectum & 7 [21.2\%] \\
\hline Mid and lower rectum & $4[12.1]$ \\
\hline \multicolumn{2}{|l|}{ Histopathology } \\
\hline Moderately differentiated & 19 [57.6\%] \\
\hline Poorly differentiated & 10 [30.3\%] \\
\hline Mucinous adenocarcinoma & 4 [12.1\%] \\
\hline \multicolumn{2}{|l|}{ Clinical stage } \\
\hline$\|$ & 10 [30.3\%] \\
\hline IIIA & 6 [18.2\%] \\
\hline IV & 17 [51.5\%] \\
\hline \multicolumn{2}{|l|}{ Tstage } \\
\hline T3 & 32 [96.96\%] \\
\hline T4 & 1 [3.03\%] \\
\hline \multicolumn{2}{|l|}{ Nstage } \\
\hline NO & 13 [39.3\%] \\
\hline N1 & 12 [36.36\%] \\
\hline N2 & 8 [24.24\%] \\
\hline \multicolumn{2}{|c|}{ FDG-PET/CT results, median, range } \\
\hline SUVmax & $17.9[5.3-53.82]$ \\
\hline SUV mean & $10.46[2.7-40.5]$ \\
\hline MTV & $21.5[3.9-62.5]$ \\
\hline TLG & $234.442[36.9-2521.1]$ \\
\hline
\end{tabular}

SUV standard uptake value, MTV metabolic tumor volume, TLG total lesion glycolysis

MTV and TLG ( $p=0.763$ and $p=0.701$, respectively) (Figs. 2, 3, 4 and Table 2).

Correlations between FDG-PET/CT parameters and Patient/Tumor Characteristics are detailed in Table 3. No correlation was found between tumor size and SUVmax $(p>0.05)$. Yet, MTV and TLG were highly correlated with the tumor size $(p<0.01)$ (Fig. 5 and Table 3). Further, PET parameters were not influenced by rectal tumor origin in the upper, middle, and lower rectum (Table 3).

\section{Metabolic characteristics in relation to clinical stage}

There was no significant correlation between the PET/ CT parameters [SUVmax, SUVmean, MTV, and TLG] and metastatic load [negative, oligo-metastases and multiple metastasis] $(p>0.05)$ (Table 3). The SUVmax $(p=0.896)$, SUVmean $(p=0.901)$, MTV $(p=0.128)$ and TLG $(p=0.336)$ values showed no statistical 


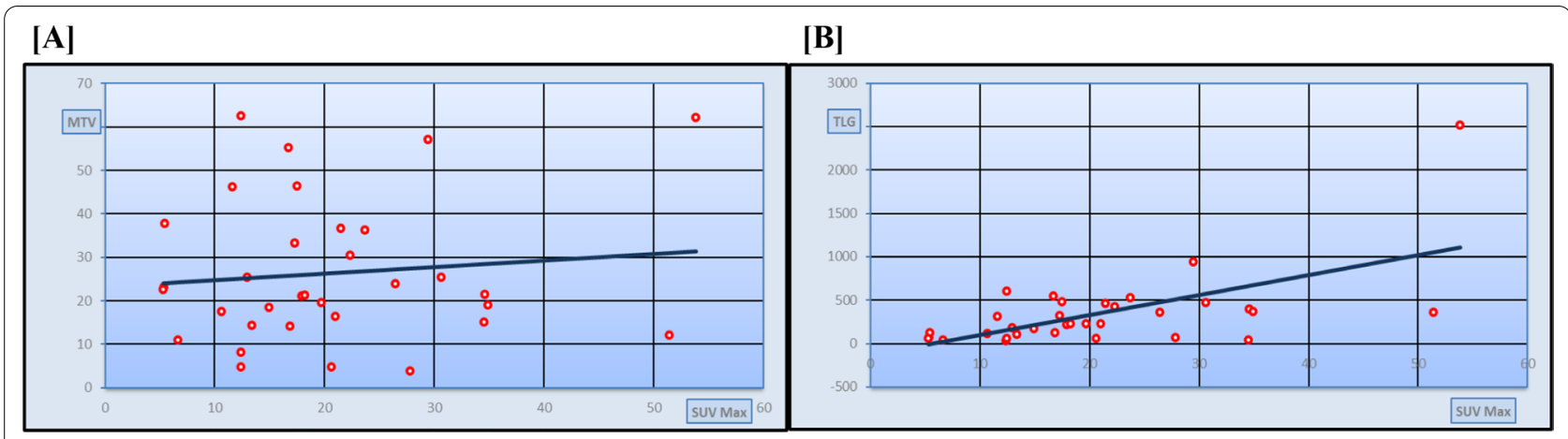

Fig. 1 The correlation between SUVmax and the metabolic parameters

Table 2 Demographic and PET/CT characteristics according to the different histopathological groups

\begin{tabular}{|c|c|c|c|c|}
\hline & Mucinous & Moderate differentiated & Poorly differentiated & $p$ value \\
\hline Total, \% & $4[12.1 \%]$ & $19[57.6 \%]$ & $10[30.3] \%$ & - \\
\hline Age $^{*}$ & $49[35.5-59.5]$ & $64[50-73]$ & $60.5[55.75-63]$ & 0.238 \\
\hline Sex, M:F & $2: 2$ & $12: 7$ & $5: 5$ & 0.752 \\
\hline Tumor size & $70[40-77.5]$ & $51[48-67]$ & $45[31.25-63.75]$ & 0.314 \\
\hline SUV max* & $12.685[8.0525-14.415]$ & $22.3[17.9-34.5]$ & 14.55 [10.0575-17.6825] & 0.003 \\
\hline SUV mean* & 8.375 [4.76-9.6575] & 13.88 [9.67-18.61] & 8.315 [5.92-10.5375] & 0.019 \\
\hline MTV $\mathrm{cm}^{3 *}$ & 21.99 [12.845-53.23] & 21.06 [15.17-30.46] & $29.475[12.7675-46.3875]$ & 0.763 \\
\hline TLG, $\mathrm{g} / \mathrm{ml}^{*} \times \mathrm{cm}^{3 *}$ & $180.822[75.219-504.527]$ & 322.398 [113.286-422.785] & $178.218[60.075-497.251]$ & 0.701 \\
\hline
\end{tabular}

SUV standard uptake value, MTV metabolic tumor volume, TLG total lesion glycolysis

*Median, range

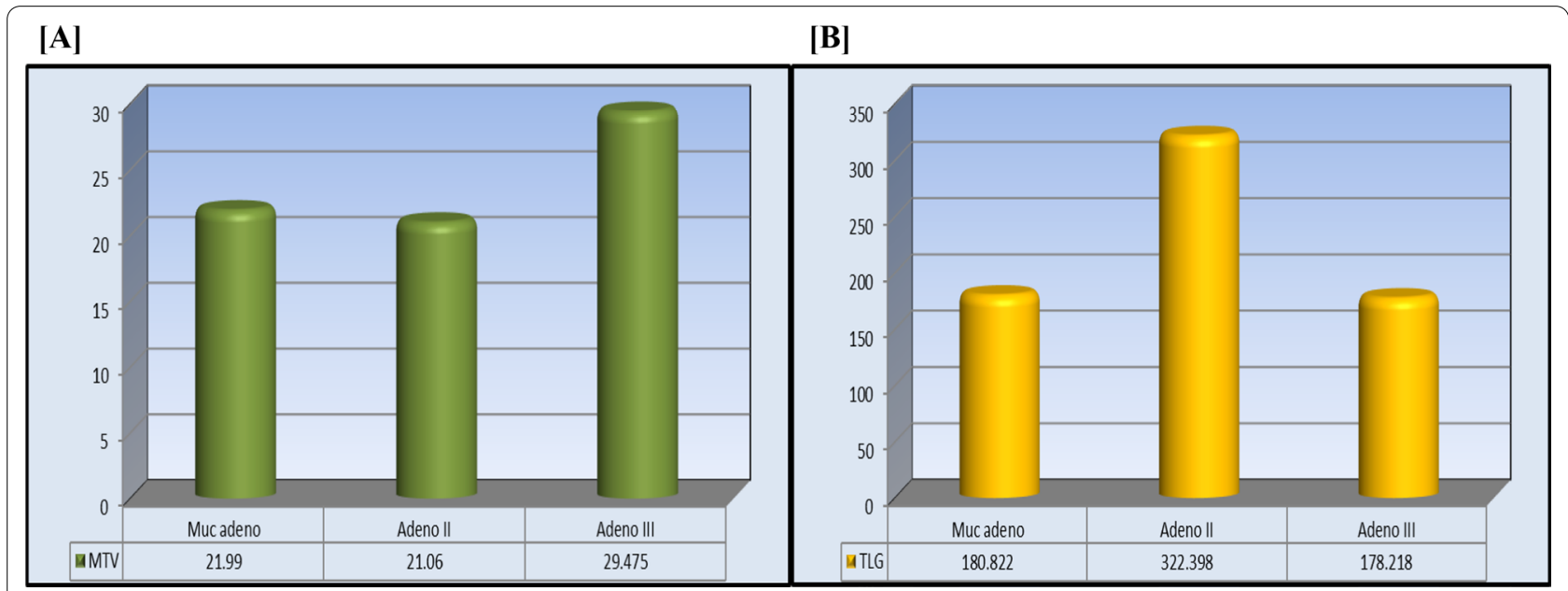

Fig. 2 The correlation between the metabolic parameters and rectal mass histopathological type

differences between the clinical stages [II, IIIA, and IV] (Table 3).

\section{Discussion}

Selection of high-risk patients with cancer rectum is crucial. Imaging tools to assess rectal cancer aggressiveness is still under investigation. The purpose of this study is to evaluate the significance of metabolic parameters 


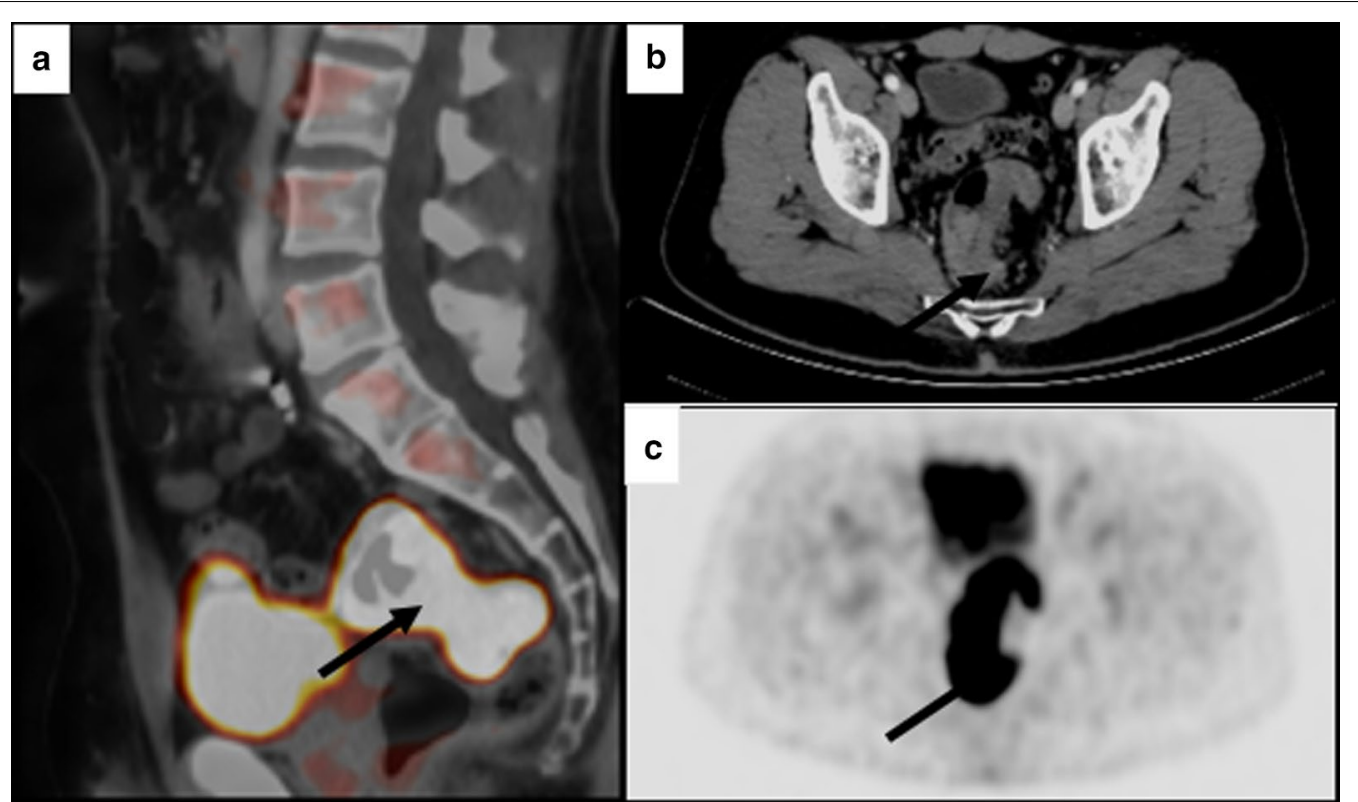

Fig. 3 44-year-old male patient with recently diagnosed rectal cancer, histopathological proven adenocarcinoma grade III. Sagittal fused PET/ CT of pelvis (a), Axial CT of the pelvis (b), axial PET of the pelvis (c) show irregular polypoidal hypermetabolic mass involving upper rectum [black arrows] with SUVmax of 23.68 , MTV of $36.35 \mathrm{~cm}^{3}$, and TLG of $528.89 \mathrm{~g} / \mathrm{ml}^{*} \times \mathrm{cm}^{3}$

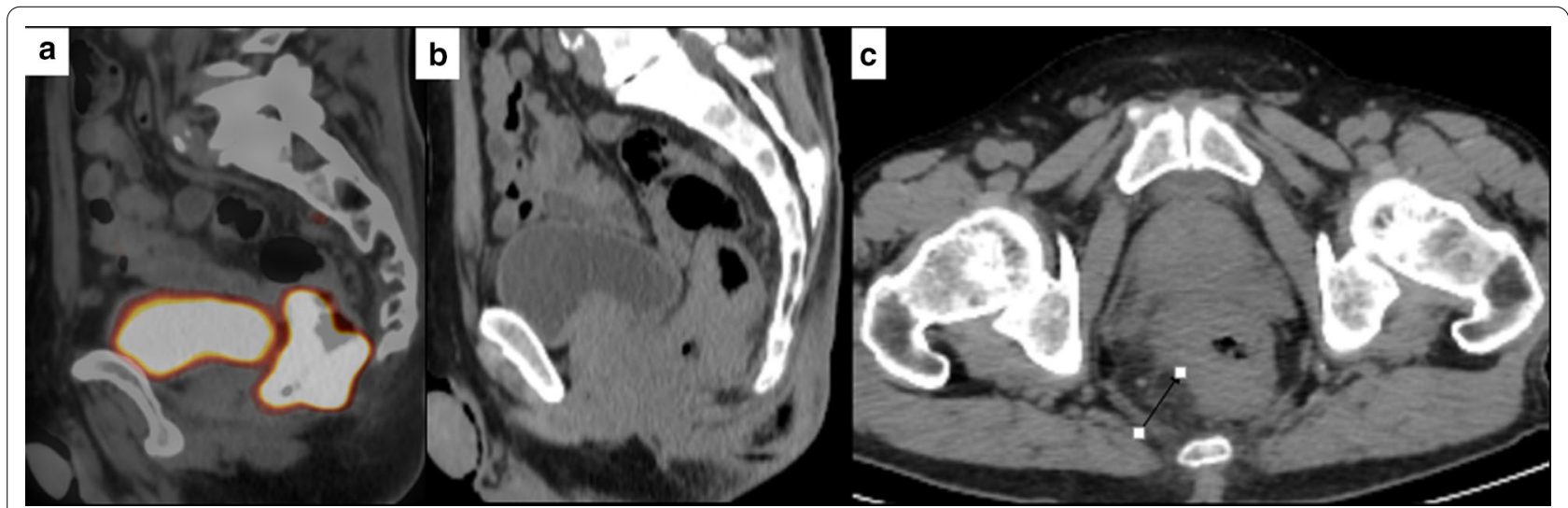

Fig. 4 67-year-old male patient with recently diagnosed rectal cancer, histopathological proven adenocarcinoma grade II. Sagittal fused PET/ CT of the pelvis (a), Sagittal CT of the pelvis (b), and axial CT (c) show metabolically active focal mural thickening at the lower rectum [thin arrow] achieving SUVmax 22.3, MTV of $30.46 \mathrm{~cm}^{3}$, and TLG of $422.7 \mathrm{~g} / \mathrm{ml}^{*} \times \mathrm{cm}^{3}$. Few small inactive perirectal lymph nodes [thick arrow]

measured by baseline PET/CT as a non-invasive tool in predicting aggressiveness of the rectal cancer.

MTV and TLG are volume-based 18F-FDG PET parameters. CT and MRI can also indicate the tumour volume. However due to the uneven shape of malignant tumors, unclear boundaries, and the presence of necrotic component, these modalities may not be reliable [13]. These volume-based 18F-FDG PET parameters may more accurately reflect viable tumour volume and grade of malignancy as MTV and TLG do not only offer volumetric tumour burden but also identify viable tumour region with metabolic information [10]. MTV is a notable prognostic factor in different diseases since it addresses the double attributes of tumor volume and the level of FDG uptake by the tumor [14]. TLG has been proposed as a more accurate parameter because it considers both SUVmean and MTV [15]. A worse prognosis is expected with high MTV and TLG [16-18]. Therefore, the incorporation of these metabolic parameters with conventional risk factors (size, histopathological type and 
Table 3 Correlations between metabolic parameters of FDG-PET/CT and patient/tumor characteristics

\begin{tabular}{|c|c|c|c|c|c|c|c|c|c|}
\hline Variables & $N$ & SUVmax* & $p$ & SUV mean* & $p$ & MTV* & $P$ & TLG* & $p$ \\
\hline Size & 33 & & 0.85 & & 0.25 & & 0.003 & & 0.003 \\
\hline Location & & & 0.33 & & 0.37 & & 0.89 & & 0.59 \\
\hline Lower ${ }^{*}$ & 19 & $12.96[6.6-22.3]$ & & 7.4 [3.88-13.88] & & 21.5 [10.94-25.4] & & $188.11[57.2-42.05]$ & \\
\hline Middle, lower & 3 & $15.4[7.36-17.8]$ & & 9.18 [4.3-10.62] & & 22.18 [16.02-40.72] & & $168.99[84.1-421.2]$ & \\
\hline middle $e^{*}$ & 7 & 20.6 [14.9-0] & & $12.9[9.4-0]$ & & $18.56[4.86-0]$ & & 173.53 [62.69-0] & \\
\hline Upper $^{*}$ & 4 & $20.99[12.41-29.43]$ & & $10.78[7.7-16.6]$ & & $23.87[15.2-37.8]$ & & $322.4[122.7-472.9]$ & \\
\hline Clinical stage & & & 0.89 & & 0.9 & & 0.13 & & 0.34 \\
\hline$\|^{*}$ & 10 & 19.445 [10.92-35.8] & & $11.68[6.1675-21.4]$ & & $18.785[10.27-28.225]$ & & $229.58[53.5-467.2]$ & \\
\hline$\| A^{*}$ & 6 & $16.98[9.32-25.45$ & & $10.4[5.7325-15.315]$ & & 18.4 [11.97-25.09] & & $114.74[71.42-387.3]$ & \\
\hline $\mathrm{IV}^{*}$ & 17 & $17.5[13.93-27.1]$ & & 9.97 [8.17-14.875] & & 25.42 [19.07-46.435] & & $315.58[150.7-507.8]$ & \\
\hline Metastasis load & & & 0.78 & & 0.9 & & 0.104 & & 0.18 \\
\hline Negative $^{*}$ & 16 & $19.25[11.07-28.525]$ & & 11.68 [6.66-17.507] & & 18.4 [11.24-24.88] & & $170.45[61.44-410.97]$ & \\
\hline Oligo-metastasis ${ }^{*}$ & 6 & $17.87[11.1-21.7]$ & & $10.61[6.4-13.4]$ & & 23.33 [15.7-39.9] & & 208.48 [109.1-298.04] & \\
\hline multiple $^{*}$ & 11 & $17.3[14.9-29.4]$ & & 9.8 [8.9-15.2] & & $33.34[18.56-55.23]$ & & $362.8[173.5-550.6]$ & \\
\hline
\end{tabular}

*Median, range

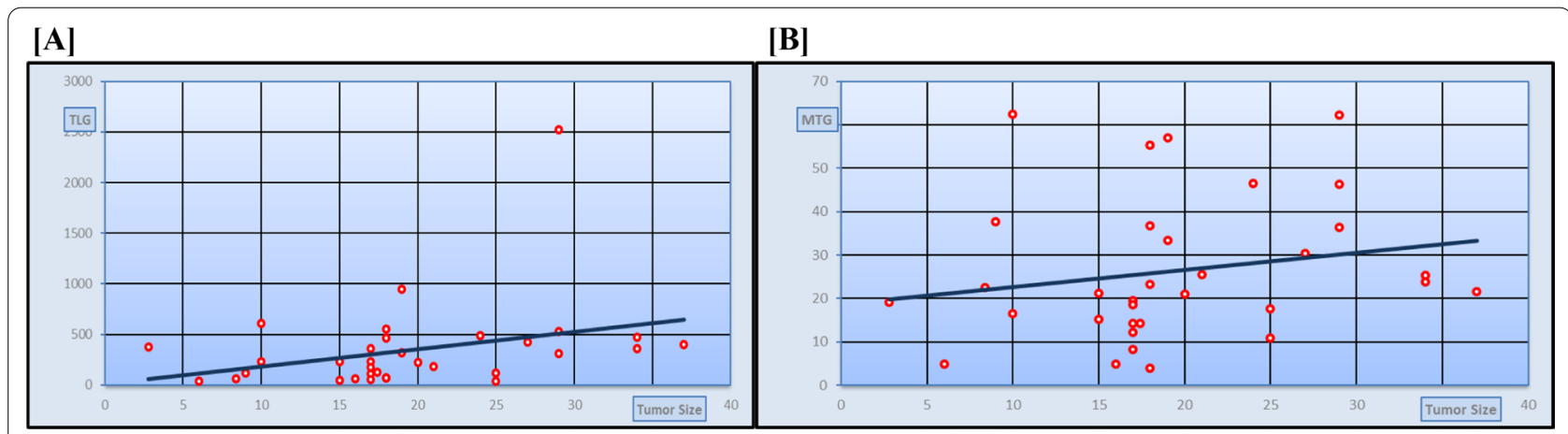

Fig. 5 The correlation between the metabolic parameters and size of the rectal mass

the tumor stage) may help to provide more accurate pretreatment risk stratification in rectal cancer patients than conventional risk factors alone.

In our study, we find no significant association between these metabolic parameters (MTV and TLG) and the clinical stage of rectal cancer. Similar findings were reported in various types of cancer. Studies conducted by Stahl et al. [19] and Kaida et al. [20] found no significant difference in FDG uptake between different tumor stages, including gastric tumor and papillary thyroid carcinoma respectively. On the contrary, Deantonio et al. [21] reported that a higher level of metabolic and volumetric parameters was significantly associated with T4 stage of rectal cancer, which is generally considered a more aggressive disease. This discordance is probably due to the relatively small number of our cases and the minimal heterogeneity regarding the radiological stage.

The present study demonstrates a strong association between the size of the primary rectal tumor and the metabolic FDG parameters [MTV and TLG]. Similarly, Kaymak et al. [22] found positive relation between colorectal tumour diameter with MTV and TLG. We find no significant correlation between PET/CT metabolic parameters [MTV and TLG] and different tumor characteristics [location, stage, and metastatic load].

Determining tumor type is crucial because different tumor types differ in terms of radio-sensitivity, local behavior, and the tendency for regional and systemic metastasis. In addition, the tumor's histological grade is a key predictor of local invasion and systemic metastasis [23].

Our study demonstrates that mucinous and non-mucinous rectal adenocarcinoma [moderate and poorly differentiated adenocarcinoma] have different 18F-FDG uptake in PET/CT imaging. SUV estimates [mean and maximum] were greater in the moderately differentiated group. Several studies investigated different 18FDG uptake between the histopathological subtypes of 
the primary rectal tumor. Horvat et al. [24] and Berger et al. [25] concluded that mucinous tumors had low 18-FDG uptake in both PET/CT and PET/MRI. Tumour FDG avidity in PET inversely correlates with the overall amount of mucin, which may result in false-negative cases. On the other hand, Dos Anjos et al. [26] demonstrated that mucinous and non-mucinous rectal cancers had similar 18F-FDG uptake in PET/CT and justifying these results by the solid components of the mucinous tumors, which appeared to be extremely FDG-avid, and may had compensated for the lack of 18F-FDG uptake of the mucinous component.

We find that the different histological subtypes of rectal tumor [MAC, moderately or poorly differentiated adenocarcinoma] show no significant difference regarding the median values of MTV and TLG. Dos Anjos et al. [26] showed that MTV and TLG were higher at the mucinous group than the non-mucinous one and explained these results by larger tumor volumes among this histological subtype. Also, our results disagree with Kaymak et al. [22]. They found significant correlation between tumor differentiation and MTV. The discordance between our results with the previous studies is probably due to a slightly higher incidence of moderately differentiated adenocarcinoma in our patients, the most common histopathological type of rectal tumor.

In contrast to some other tumor types, our study demonstrates that moderately differentiated rectal cancer [grade 2] accumulates FDG more intensely than does poorly differentiated one [grade 3]. Similar results have been reported in tumors other than rectal cancer. Study conducted by Stahl et al. [19] found an inverse relation between gastric tumour FDG uptake and the degree of tumour differentiation (grading). In addition, in a study done by Song et al. [27], moderately differentiated gastroesophageal cancer had higher metabolic parameters than poorly differentiated lesions. In rectal carcinomas, the degree of tumour differentiation is not a proven prognostic factor and may only be of a predictive value [28]. Therefore, grading may not represent biological aggressiveness in this tumour entity.

There are some limitations of our study. The retrospective nature of the study in a single medical center may have increased the risk of selection bias. Tumor size is measured in CT images yet, measuring tumor size in T2-weighted MRI images is more reliable. The inclusion of small tumors $(1-2 \mathrm{~cm})$ in our series may have affected estimation of SUV and MTV values by partial volume effect, as suggested in previous studies [29]. We are unable to link our finding with the patients' progressionfree survival or overall survival due to a lack of adequate patient follow-up. The important consideration is the small sample size, and this is due to the high cost of the technique. Future trials with a larger number of patients may be needed to obtain more accurate result.

\section{Conclusion}

We conclude that using FDG PET/CT as a baseline investigation in rectal tumor has a limited role in the selection of high-risk patients with aggressive disease who require more intensive treatment and follow-up schedules.

\section{Abbreviations \\ [(18F) FDG-PET/CT]: Fluorodeoxyglucose positron emission tomography/ computed tomography; SUVmax: Maximum mean standard uptake values; SUV mean: Mean standard uptake values; MTV: Mean tumor volume; TLG: Total lesion glycolysis; MAC: Mucinous adenocarcinoma; CRC: Colorectal cancer; ROI: Region of interest.}

\section{Acknowledgements}

We would like to thank Dr. Yehia Omar from Misr Radiology Center for his help in collecting the cases.

\section{Authors' contributions}

All authors contributed to the study conception and design. Material preparation, data collection and analysis were performed by Iman Sherif, and Rasha S. Hussein. The first draft of the manuscript was written by Iman Sherif and all authors commented on previous versions of the manuscript. All authors read and approved the final manuscript.

\section{Funding}

This research did not receive any specific grant from funding agencies in the public, commercial, or not-for-profit sectors.

\section{Availability of data and materials}

The datasets used and analyzed during the current study are available from the corresponding author on reasonable request.

\section{Declarations}

\section{Ethics approval and consent to participate}

The study was performed after approval of the Ethical Committee of Scientific Research, Faculty of Medicine, and Ain Shams University. Consent was taken from all patients.

\section{Consent for publication}

All patients included in this research gave written informed consent to publish the data contained within this study.

\section{Competing interests}

The authors have no relevant financial or non-financial interests to disclose. The authors have no conflicts of interest to declare that are relevant to the content of this article.

Received: 21 September 2021 Accepted: 9 November 2021

Published online: 15 November 2021

References

1. Bray F, Ferlay J, Soerjomataram I, Siegel RL, Torre LA, Jemal A (2018) Global cancer statistics 2018: GLOBOCAN estimates of incidence and mortality worldwide for 36 cancers in 185 countries. CA Cancer J Clin 68:394-424. https://doi.org/10.3322/caac.21492

2. Rödel C, Martus P, Papadoupolos T, Füzesi L, Klimpfinger M, Fietkau R et al (2005) Prognostic significance of tumor regression after preoperative chemoradiotherapy for rectal cancer. J Clin Oncol 23:8688-8696. https:// doi.org/10.1200/JCO.2005.02.1329 
3. Valentini V, Coco C, Picciocchi A, Morganti AG, Trodella L, Ciabattoni A et al (2002) Does downstaging predict improved outcome after preoperative chemoradiation for extraperitoneal locally advanced rectal cancer? A long-term analysis of 165 patiants. Int J Radiat Oncol Biol Phys 53:664-674. https://doi.org/10.1016/50360-3016(02)02764-5

4. Hancerliogullari O, Okuyucu K, Ince S, Peker S, Arslan N (2020) Prognostic parameters in recurrent colorectal cancer: A role of control or restaging by FDG-PET/CT. Vojnosanit Pregl 77:201-208. https://doi.org/10.2298/ VSP170817065H

5. Chiu KWH, Lam KO, An H, Cheung GTC, Lau JKS, Choy TS, Lee VHF (2018) Long-term outcomes and recurrence pattern of 18F-FDG PET-CT complete metabolic response in the first-line treatment of metastatic colorectal cancer: a lesion-based and patient-based analysis. BMC Cancer 18:1-10. https://doi.org/10.1186/s12885-018-4687-9

6. Jadvar H, Alavi A, Gambhir S (2009) 18F-FDG uptake in lung, breast, and colon cancers: molecular biology correlates and disease characterization. J Nucl Med 50:1820-1827. https://doi.org/10.2967/jnumed.108.054098

7. Hatt M, Visvikis D, Albarghach NM, Tixier F, Pradier O, Cheze-le Rest C (2011) Prognostic value of 18 F-FDG PET image-based parameters in oesophageal cancer and impact of tumour delineation methodology. Eur J Nucl Med Mol Imaging 38:1191-1202. https://doi.org/10.1007/ s00259-011-1755-7

8. Hyun SH, Choi JY, Shim YM, Kim K, Lee SJ, Cho YS et al (2010) Prognostic value of metabolic tumor volume measured by $18 \mathrm{~F}$-fluorodeoxyglucose positron emission tomography in patients with esophageal carcinoma. Ann Surg Oncol 17:115-122. https://doi.org/10.1245/s10434-009-0719-7

9. Zhang C, Chen Y, Xue H, Zheng P, Tong J, Liu J, Sun X, Huang G (2009) Diagnostic value of FDG-PET in recurrent colorectal carcinoma: a metaanalysis. Int J Cancer 124:167-173. https://doi.org/10.1002/ijc.23926

10. Choi BW, Kang S, Bae SU, Jeong WK, Baek SK, II Song B, Won KS, Kim HW (2021) Prognostic value of metabolic parameters on 18F-fluorodeoxyglucose positron tomography/computed tomography in classical rectal adenocarcinoma. Sci Rep 11:1-16. https://doi.org/10.1038/ s41598-021-92118-X

11. Shi D, Cai G, Peng J, Li D, Li X, Xu Y, Cai S (2015) The preoperative SUVmax for 18 F-FDG uptake predicts survival in patients with colorectal cancer. BMC Cancer 15:1-8. https://doi.org/10.1186/s12885-015-1991-5

12. Lee JE, Kim SW, Kim JS, Choi KY, Kang WK, Oh ST et al (2012) Prognostic value of 18-fluorodeoxyglucose positron emission tomography-computed tomography in resectable colorectal cancer. World J Gastroenterol WJG 18:5072-5077. https://doi.org/10.3748/wjg.v18.i36.5072

13. Ogawa S, Itabashi M, Kondo C, Momose M, Sakai S, Kameoka S (2015) Prognostic value of total lesion glycolysis measured by $18 \mathrm{~F}-\mathrm{FDG}-\mathrm{PET} / \mathrm{CT}$ in patients with colorectal cancer. Anticancer Res 35:3495-3500

14. Chung MK, Jeong HS, Park SG, Jang JY, Son YI, Choi JY, Hyun SH, Park K, Ahn MJ, Ahn YC (2009) Metabolic tumor volume of [18F]-fluorodeoxyglucose positron emission tomography/computed tomography predicts short-term outcome to radiotherapy with or without chemotherapy in pharyngeal cancer. Clin Cancer Res 15:5861-5868. https://doi.org/10. 1158/1078-0432.ccr-08-3290

15. Larson SM, Erdi Y, Akhurst T, Mazumdar M, Macapinlac HA, Finn RD, Casilla C et al (1999) Tumor treatment response based on visual and quantitative changes in global tumor glycolysis using PET-FDG imaging: the visual response score and the change in total lesion glycolysis. Clin Positron Imaging 2:159-171. https://doi.org/10.1016/s1095-0397(99)00016-3

16. Pak K, Cheon GJ, Nam HY, Kim SJ, Kang KW, Chung JK, Kim EE, Lee DS (2014) Prognostic value of metabolic tumor volume and total lesion glycolysis in head and neck cancer: a systematic review and meta-analysis. J Nucl Med 55:884-890. https://doi.org/10.2967/jnumed.113.133801

17. Ulaner GA, Eaton A, Morris PG, Lilienstein J, Jhaveri K, Patil S, Fazio M, Larson S, Hudis CA, Jochelson MS (2013) Prognostic value of quantitative fluorodeoxyglucose measurements in newly diagnosed metastatic breast cancer. Cancer Med 2:725-733. https://doi.org/10.1002/cam4.119

18. Grabinska K, Pelak M, Wydmanski J, Tukiendorf A, d'Amico A (2015) Prognostic value and clinical correlations of 18-fluorodeoxyglucose metabolism quantifiers in gastric cancer. World J Gastroenterol WJG 21:5901

19. Stahl A, Ott K, Weber WA, Becker K, Link T, Siewert J, Schwaiger M, Fink U (2003) Original article FDG PET imaging of locally advanced gastric carcinomas : correlation with endoscopic and histopathological findings. Eur J Nucl Med Mol Imaging 30:288-295. https://doi.org/10.1007/ s00259-002-1029-5

20. Kaida H, Kawahara A, Hayakawa M, Hattori S, Kurata S, Fujimoto K, Azuma K, Hirose Y, Takamori S, Hiromatsu Y, Nakashima T, Fujita H et al (2014) The difference in relationship between 18 F-FDG uptake and clinicopathological factors on thyroid, esophageal, and lung cancers. Nucl Med Commun 35:36-43. https://doi.org/10.1097/mnm.0000000000000019

21. Deantonio L, Caroli A, Puta E, Ferrante D, Apicella F, Turri L, Sacchetti G, Brambilla M, Krengli M (2018) Does baseline [18F] FDG-PET/CT correlate with tumor staging, response after neoadjuvant chemoradiotherapy, and prognosis in patients with rectal cancer? Radiat Oncol 13:1-9. https://doi. org/10.1186/s13014-018-1154-3

22. Kaymak ZA, Karahan N, Erdoğan M, Erdemoğlu E, Zihni I, Şengül SS (2021) Correlation of 18F-FDG/PET SUVmax, SUVmean, MTV, and TLG with HIF-1a in patients with colorectal cancer. Mol Imaging Radionucl Ther 30(2):93-100. https://doi.org/10.4274/mirt.galenos.2021.04934

23. Nabi U, Nagi AH, Riaz S, Sami W (2010) Morphological evaluation of colorectal carcinoma with grading staging and histological types. JPMAJournal Pakistan Med Assoc 60:998

24. Horvat N, Hope TA, Pickhardt PJ, Petkovska I (2019) Mucinous rectal cancer: concepts and imaging challenges. Abdom Radiol 44:3569-3580. https://doi.org/10.1007/s00261-019-02019-x

25. Berger KL, Nicholson SA, Dehdashti F, Siegel BA (2000) FDG PET evaluation of mucinous neoplasms: correlation of FDG uptake with histopathologic features. Am J Roentgenol 174:1005-1008. https://doi.org/10.2214/ ajr.174.4.1741005

26. dos Anjos DA, Habr-Gama A, Vailati BB, Rossi CB, Coturel AE, Perez RO, São Julião GP, de Sousa JB, Buchpiguel CA (2016) 18F-FDG uptake by rectal cancer is similar in mucinous and nonmucinous histological subtypes. Ann Nucl Med 30:513-517. https://doi.org/10.1007/s12149-016-1089-4

27. Song J, Li Z, Chen P, Zhou N, Zhang Y, Yang Z, Wang X (2020) The correlation between molecular pathological profiles and metabolic parameters of 18 F-FDG PET/CT in patients with gastroesophageal junction cancer. Abdom Radiol 45:312-321. https://doi.org/10.1007/s00261-019-02065-5

28. Compton CC, Fielding LP, Burgart LJ, Cooper HS, Conley B et al (1999) Prognostic factors in colorectal cancer. College of American Pathologists Consensus Statement. Arch Pathol Lab Med 124:979-994. https://doi.org/ 10.5858/2000-124-0979-pficc

29. Soret BSL, Buvat I (2007) Partial-volume effect in PET tumor imaging. J Nucl Med 48:932-945. https://doi.org/10.2967/jnumed.106.035774

\section{Publisher's Note}

Springer Nature remains neutral with regard to jurisdictional claims in published maps and institutional affiliations. 